\title{
Dural Arteriovenous Fistula Following Translabyrinthine Resection of Cerebellopontine Angle Tumors: Report of Two Cases
}

\author{
Peter M.M.C. Li, M.D., ${ }^{1}$ Nancy J. Fischbein, M.D., ${ }^{1,2}$ Huy M. Do, M.D., 2,3 \\ and Nikolas H. Blevins, M.D. ${ }^{1}$
}

We describe two cases of dural arteriovenous fistula (DAVF) developing in a delayed fashion after translabyrinthine resection of cerebellopontine angle tumors. Two patients in an academic tertiary referral center, a 46-year-old woman and a 67-year-old man, underwent translabyrinthine resection of a $2-\mathrm{cm}$ left vestibular schwannoma and a $4-\mathrm{cm}$ left petrous meningioma, respectively. Both patients subsequently developed DAVF, and in each case the diagnosis was delayed despite serial imaging follow-up. In one patient, cerebrospinal fluid diversion before DAVF was identified as the cause of her intracranial hypertension; the other patient was essentially asymptomatic but with a high risk of hemorrhage due to progression of cortical venous drainage. Endovascular treatment was effective but required multiple sessions due to residual or recurrent fistulas. Dural arteriovenous fistula is a rare complication of translabyrinthine skull base surgery. Diagnosis requires a high index of clinical suspicion and an understanding of subtle imaging findings that may be present on follow-up studies performed for tumor surveillance. Failure to recognize this complication may lead to misguided interventions for treatment of hydrocephalus and other complications, as well as ongoing risks related to venous hypertension and intracranial hemorrhage. As this condition is generally curable with neurointerventional and/or surgical methods, timely diagnosis and treatment are essential.

KEYWORDS: Dural arteriovenous fistula, translabyrinthine surgery, skull base, embolization

Results from resection of cerebellopontine angle (CPA) tumors are significantly affected by variations in adjacent neurovascular anatomy. ${ }^{1,2}$ Despite an overall improvement in patient outcomes, vascular complications continue to be reported in up to $7 \%$ of patients undergoing CPA tumor resection. ${ }^{3}$ Due to the signifi- cant potential morbidity and mortality associated with vascular injury, meticulous surgical technique is required to remove the tumor while minimizing trauma to adjacent vasculature.

Vascular damage can result in acute hemorrhagic or ischemic complications. Injury to the transverse and
${ }^{1}$ Departments of Otolaryngology, Head and Neck Surgery; ${ }^{2}$ Radiology and ${ }^{3}$ Neurosurgery, Stanford University Medical Center, Stanford, California.

Address for correspondence and reprint requests: Peter M.M.C. Li, M.D., 801 Welch Road, Stanford, CA 94305 (e-mail: peteli@ stanford.edu).

Skull Base Rep 2011;1:51-58. Copyright (C) 2011 by Thieme
Medical Publishers, Inc., 333 Seventh Avenue, New York, NY 10001, USA. Tel: +1(212) 584-4662.

Received: August 22, 2010. Accepted: January 10, 2011. Published online: April 4, 2011.

DOI: http://dx.doi.org/10.1055/s-0031-1275634.

ISSN 2157-6971. 
sigmoid sinuses may lead to thrombosis. Due to venous redundancy, unilateral dural sinus occlusion may remain asymptomatic, although complications of venous insufficiency can occur. ${ }^{4}$

In the acute setting, venous occlusion can cause cerebral edema, brain herniation, and death, whereas more indolent chronic venous insufficiency may result in increased intracranial pressure and the clinical syndrome of pseudotumor cerebri. Dural sinus thrombosis is also considered a potential precursor to the development of a dural arteriovenous fistula (DAVF), a rare vascular complication of CPA surgery. ${ }^{5}$ We present two cases in which a DAVF developed as the result of sigmoid sinus thrombosis following translabyrinthine tumor resection.

\section{CASE 1}

A 46-year-old woman presented with left-sided tinnitus and severe to profound hearing loss. Magnetic resonance imaging (MRI) showed an enhancing $23 \times 23 \times 21$-mm left-sided CPA mass consistent with vestibular schwannoma (VS; Fig. 1). Vascular anatomy appeared normal, and there was no history of coagulopathy.

A translabyrinthine resection of the VS was performed. The sigmoid sinus was exposed in a routine manner and gently retracted with a self-retaining ribbon retractor. There was no apparent intraoperative vascular injury.

Postoperatively, the patient experienced persistent headache, head pressure, and balance difficulties consistent with a peripheral deafferentation vestibulopathy. There were no signs of central vestibular dysfunction. Follow-up MRI scans at 3 and 12 months postoperatively were interpreted as showing expected postresection changes without explanation for the patient's persistent symptoms (Fig. 2). Though no gross hydrocephalus was present on imaging studies, mild hydrocephalus was considered to be a potential etiology of her symptoms, and a ventriculoperitoneal (VP) shunt was placed. After placement of the VP shunt, the patient's headache and balance difficulties improved significantly.

An MRI 2 years postresection and 2 months postVP shunt showed striking enlargement and tortuosity of cortical veins in the middle and posterior cranial fossae (Fig. 3). Abnormal serpentine flow voids were seen in the basal cisterns and overlying the cerebellar hemispheres and temporal lobes, strongly suggestive of a DAVF.

Cerebral angiography confirmed the diagnosis of a left transverse sinus DAVF fed by multiple external carotid and internal carotid branches (Fig. 4). The most prominent arterial feeders included the occipital and posterior meningeal arteries bilaterally and the left meningohypophyseal trunk. The left transverse sinus,

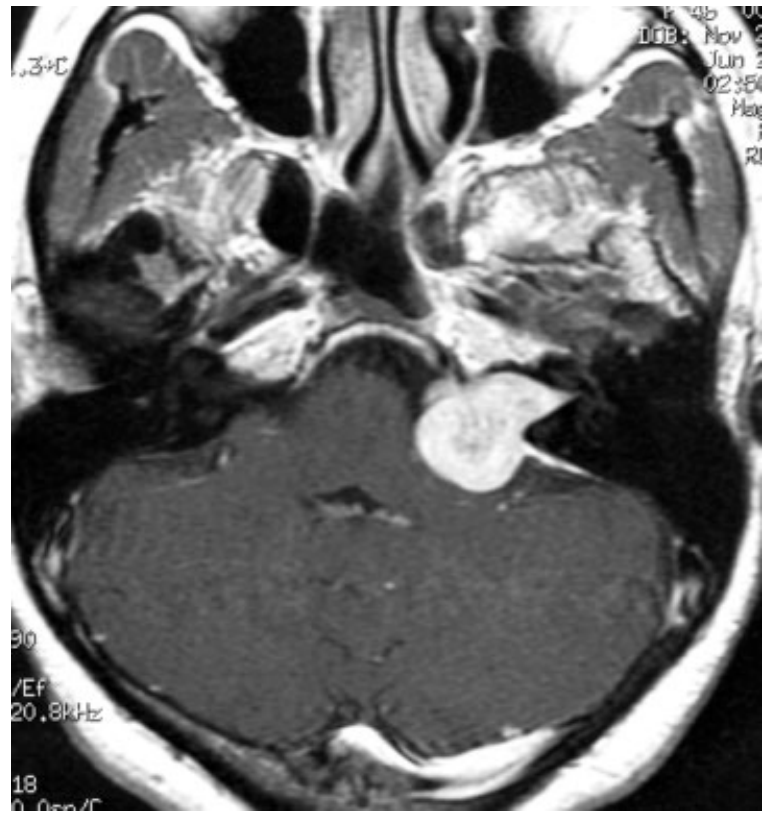

Figure 1 Patient 1: Preoperative magnetic resonance image. An axial T1-weighted image with contrast shows a classic expansile internal auditory canal and cerebellopontine angle mass consistent with left vestibular schwannoma. No vascular abnormalities are appreciated.

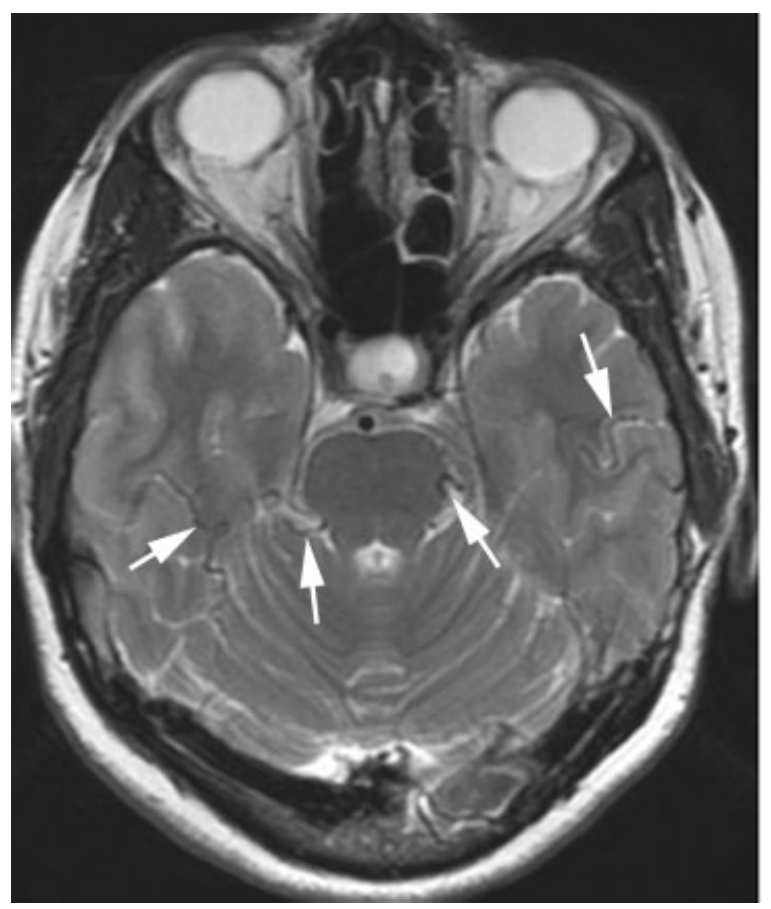

Figure 2 Patient 1: Magnetic resonance image acquired at 1 year postresection. An axial fast spin echo T2-weighted image shows multiple serpiginous hypointense structures (white arrows) consistent with venous flow voids in the basal cisterns and overlying the cerebral hemispheres. These were not noted prospectively but are definitely abnormal and represent abnormally dilated and tortuous cortical veins. 


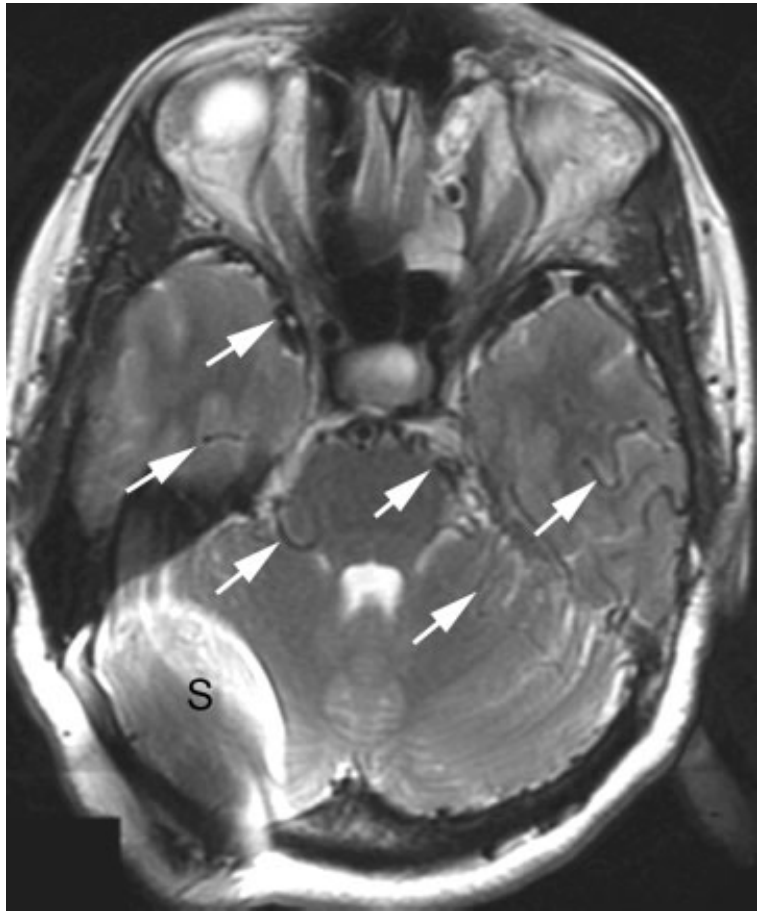

Figure 3 Patient 1: Magnetic resonance image acquired at 2 years postresection. An axial T2-weighted image shows marked interval progression of abnormal serpiginous vessels consistent with dilated veins in the basal cisterns and overlying the cerebellar hemispheres and temporal lobes (white arrows). This image was acquired after the patient received a ventriculoperitoneal shunt. S, susceptibility artifact due to shunt hardware.

sigmoid sinus, and internal jugular vein were occluded, with retrograde flow into the cortical veins and right sigmoid sinus, as well as globally delayed venous drainage.

Transvenous coil embolization obliterated the fistula. The patient did well initially, but experienced severe headaches 3 months later. A diagnostic cerebral angiogram showed interval development of a new DAVF, adjacent to the previously embolized fistula and fed by the middle meningeal artery. Transarterial embolization of the middle meningeal artery resulted in fistula obliteration. The patient's symptoms improved, and a recent angiogram obtained more than 2 years following the initial embolization shows no evidence of recurrence of either fistula.

\section{CASE 2}

A 67-year-old man presented with a 20-year history of progressive left hearing loss, progressing to complete deafness over the past 3 years. He had no other neurological symptoms or signs. MRI showed a $26 \times 35 \times 43$ $\mathrm{mm}$ homogeneously enhancing, dural-based mass arising from the posterior petrous face with extension into the left internal auditory canal, consistent with menin- gioma (Fig. 5). There was moderate compression of the brain stem, but no obstructive hydrocephalus. The preoperative MRI was otherwise negative, with no evidence of a vascular malformation.

Translabyrinthine resection was performed. The sigmoid sinus was widely exposed and gentle retraction of the sigmoid sinus and posterior fossa dura was performed. A small laceration of the sinus was repaired with 6-0 prolene sutures prior to closure.

A follow-up MRI acquired 2 years postresection showed bilateral tortuous, corkscrew-like flow voids consistent with venous collaterals over the cerebral convexities and traversing the basilar cisterns (Fig. 6). There was an absence of flow void in the left transverse and sigmoid sinuses. A DAVF was suspected, but because the patient was asymptomatic, he declined angiography until a follow-up MRI 6 months later demonstrated progression of abnormal cortical venous drainage (Fig. 7).

Cerebral angiography confirmed the diagnosis of a left transverse sinus DAVF that was supplied by multiple branches of the left external carotid artery (Fig. 8). The most prominent contributions came from the occipital, ascending pharyngeal, and middle meningeal arteries, with minor contributions from the left meningohypophyseal trunk, inferolateral trunk, and right occipital and middle meningeal arteries. The left transverse sinus, sigmoid sinus, and jugular vein were occluded. There was retrograde flow into cortical veins, superior sagittal sinus, and contralateral sigmoid sinus, with delayed venous drainage globally.

Partial arterial and venous embolization was attempted, with persistence of the fistula on angiography. Therefore, a combined surgical-endovascular approach was undertaken. A left occipital-suboccipital craniotomy was used to provide access to the left transverse sinus and endovascular coils were deployed, with additional coil embolization of the occipital artery. Follow-up MRI demonstrated marked regression of the DAVF, but an angiogram 6 months postembolization demonstrated fistula recurrence, and further intervention is planned.

\section{DISCUSSION}

DAVFs were rarely identified before 1960. In the 1970s, these lesions were increasingly reported due to advances in angiography. ${ }^{6} \mathrm{~A}$ DAVF is defined as an artery-to-vein shunt in the dura mater without a discrete nidus or an intervening capillary bed. DAVF is also a dynamic process. In the early stages, venous outflow may be relatively unobstructed, but progression over time may result in retrograde flow and eventually cortical venous drainage. ${ }^{7}$ This results in elevated venous pressures, with attendant risk of intracranial hemorrhage and other complications of venous hypertension. 
A
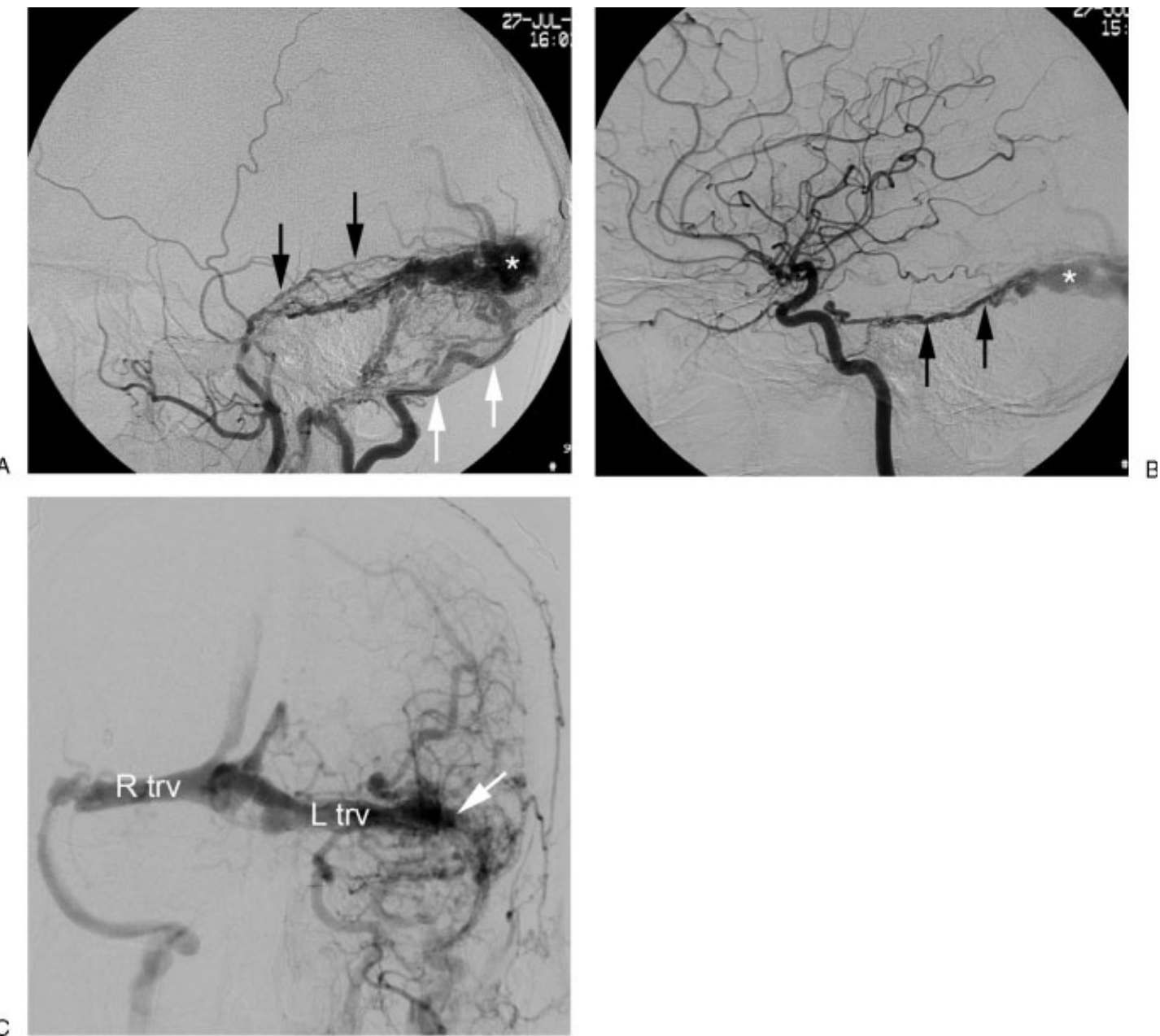

Figure 4 Patient 1: A catheter angiogram at 2 years postresection. (A) A lateral view from a left external carotid artery (ECA) injection demonstrates an abnormally enlarged occipital artery (white arrows) and abnormally enlarged posterior meningeal artery (black arrows). Multiple smaller serpentine-like vessels are seen converging on the distally occluded left transverse sinus $\left({ }^{*}\right)$. These vessels constitute ECA feeders of the dural arteriovenous fistula (DAVF). (B) A lateral view from a left internal carotid artery (ICA) injection demonstrates an abnormally enlarged and irregular meningohypophyseal trunk (black arrows) feeding the fistula $\left(^{*}\right)$. (C) An anteroposterior view from a later time (venous phase) in the left ICA injection demonstrates a tangle of vessels in the region of the distal left transverse sinus $(L \mathrm{trv})$, consistent with extensive cortical venous drainage of the DAVF. There is also distal occlusion of the left transverse sinus (white arrow). Opacified blood in the left transverse sinus drains across the torcular herophili into the right transverse sinus ( $\mathrm{R}$ trv), and then to the right sigmoid sinus and internal jugular vein.

DAVFs vary greatly in size, location, and drainage patterns, and hence their clinical presentation is highly variable. They may be asymptomatic or may cause pulsatile tinnitus, ocular symptoms, headache, vertigo, seizures, focal neurological deficits, increased intracranial pressure, or hemorrhage. DAVF involving the transverse and sigmoid sinuses is the most common type encountered by the neurotologist, as these patients often present with pulsatile tinnitus. ${ }^{7}$ Although one of our patients reported nonspecific symptoms of headache, pressure sensation, and balance difficulties, the other was surprisingly asymptomatic despite extensive and progressive cortical venous drainage.

Several postulations exist regarding the pathogenesis of DAVFs. They may be congenital or acquired, but most are acquired, often in association with venous sinus thrombosis. A hypercoagulable state, vascular manipulation, and/or trauma are potential triggers. ${ }^{8}$ Several hypotheses have been suggested to explain the development of postsurgical DAVFs. Sakaki et al presented a series of five postsurgical dural fistulae, in which histopathologic examinations of resected DAVFs showed subintimal fibrous thickening and hypertrophy of the sinus wall, as well as numerous intramural dural vessels. ${ }^{9}$ Thrombus was seen in only two of five cases. It was suggested that sigmoid sinus sacrifice resulted in intrasinus hypertension, which then induced DAVF formation. Concurrent arterial and venous injury during craniotomy may also result in DAVF. ${ }^{10}$ Desal et al suggested that venous disruption may induce angiogenesis and create an arteriovenous shunt in the dural sinus wall. ${ }^{11}$ 


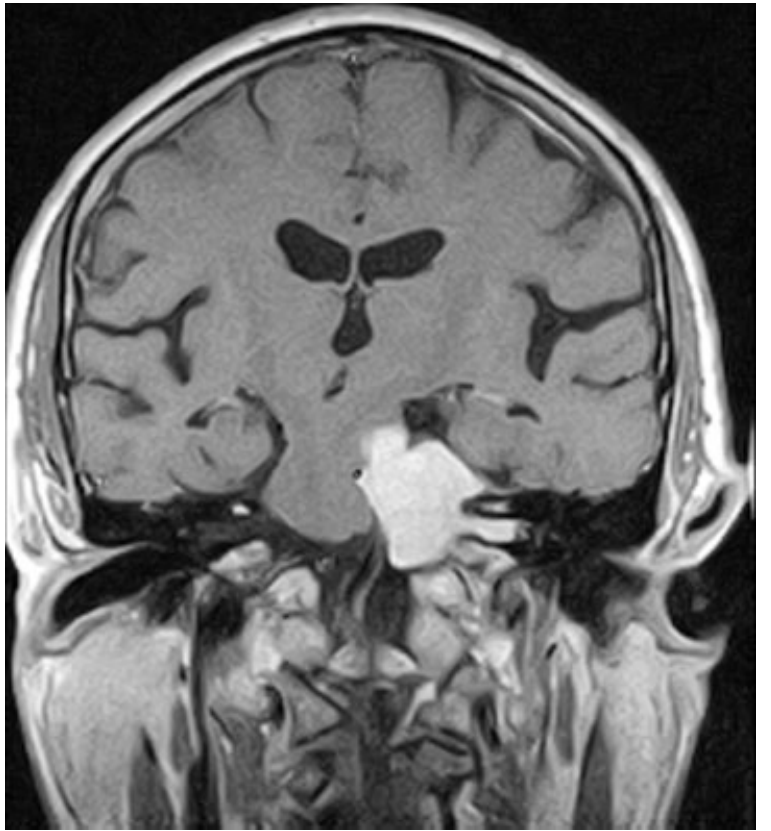

Figure 5 Patient 2: Preoperative magnetic resonance image. A coronal T1-weighted image with contrast shows a homogeneously enhancing left cerebellopontine angle mass, extending into but not enlarging the left internal carotid artery, consistent with a petrous meningioma. There are no vascular abnormalities present.

The transverse and sigmoid sinuses are at particular risk in neurotologic surgery. Occlusion may be intentional or may result from mechanical, thermal, compressive, or desiccation injury. ${ }^{12}$ Given the routine

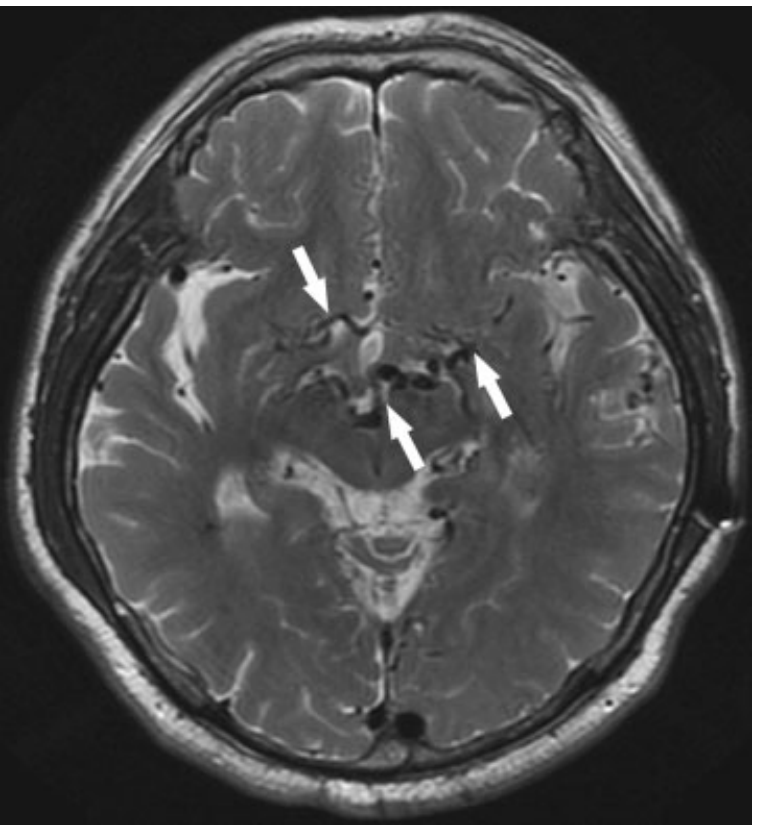

Figure 7 Patient 2: Magnetic resonance image acquired at 2.5 years postresection. An axial fast spin echo T2-weighted image shows progression of abnormal veins, with a marked increase in caliber and tortuosity. This is particularly evident around the cerebral peduncles and in the interpeduncular fossa (white arrows)

manipulation of the sigmoid sinus during translabyrinthine approaches, it is somewhat surprising that DAVFs are not more common. A search of the medical literature revealed a similar report of this complication identified

A
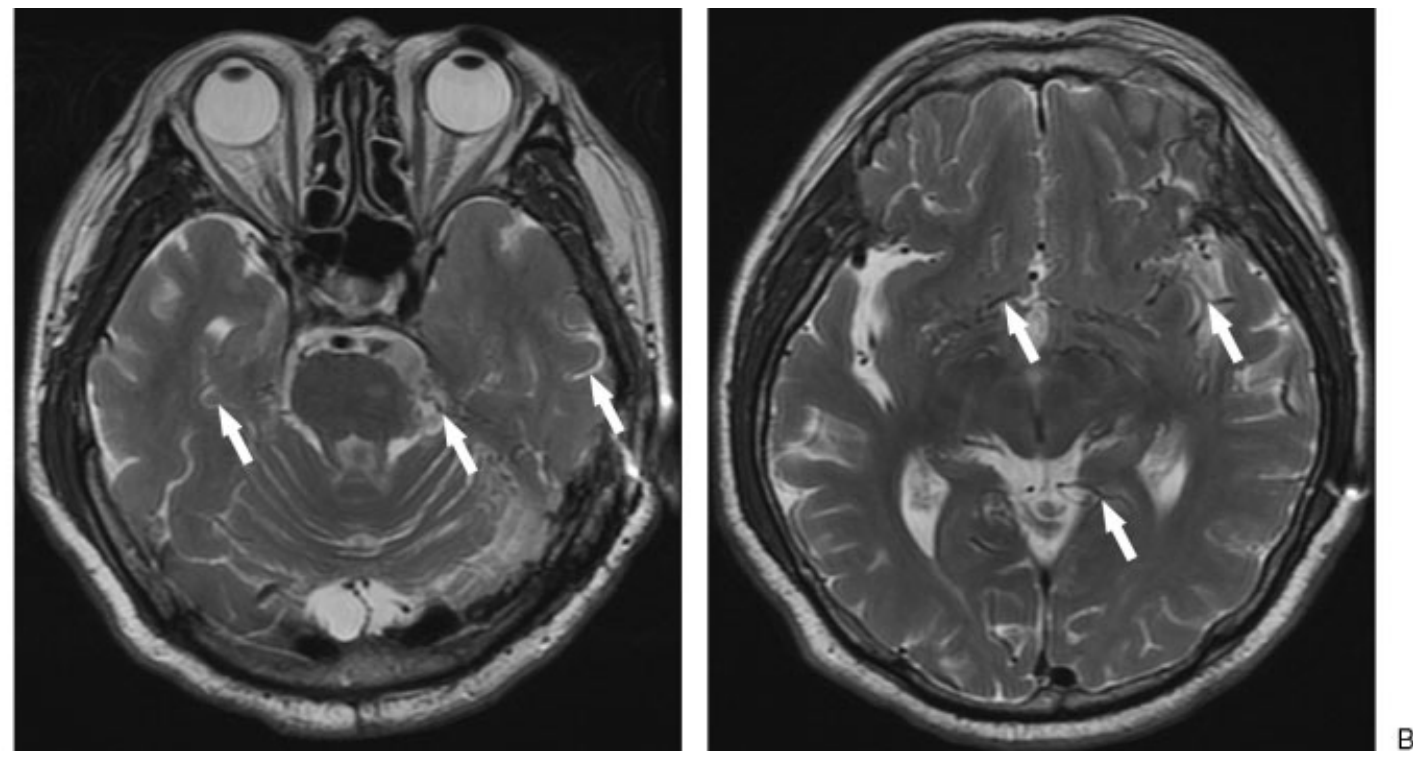

Figure 6 Patient 2: Magnetic resonance image acquired at 2 years postresection. (A) An axial fast spin echo (FSE) T2weighted image shows abnormally prominent, dilated, and tortuous cortical veins overlying the cerebellar and cerebral hemispheres, as well as present in the basal cisterns (white arrows). (B) A more superior axial FSE T2-weighted image from the same study show abnormally numerous, tortuous, and dilated veins more superiorly at the base of the brain, in the sylvian fissures, and overlying the cerebral hemispheres (white arrows). This finding was interpreted as strongly suggestive of dural arteriovenous fistula, but the patient initially refused angiographic evaluation. 
A
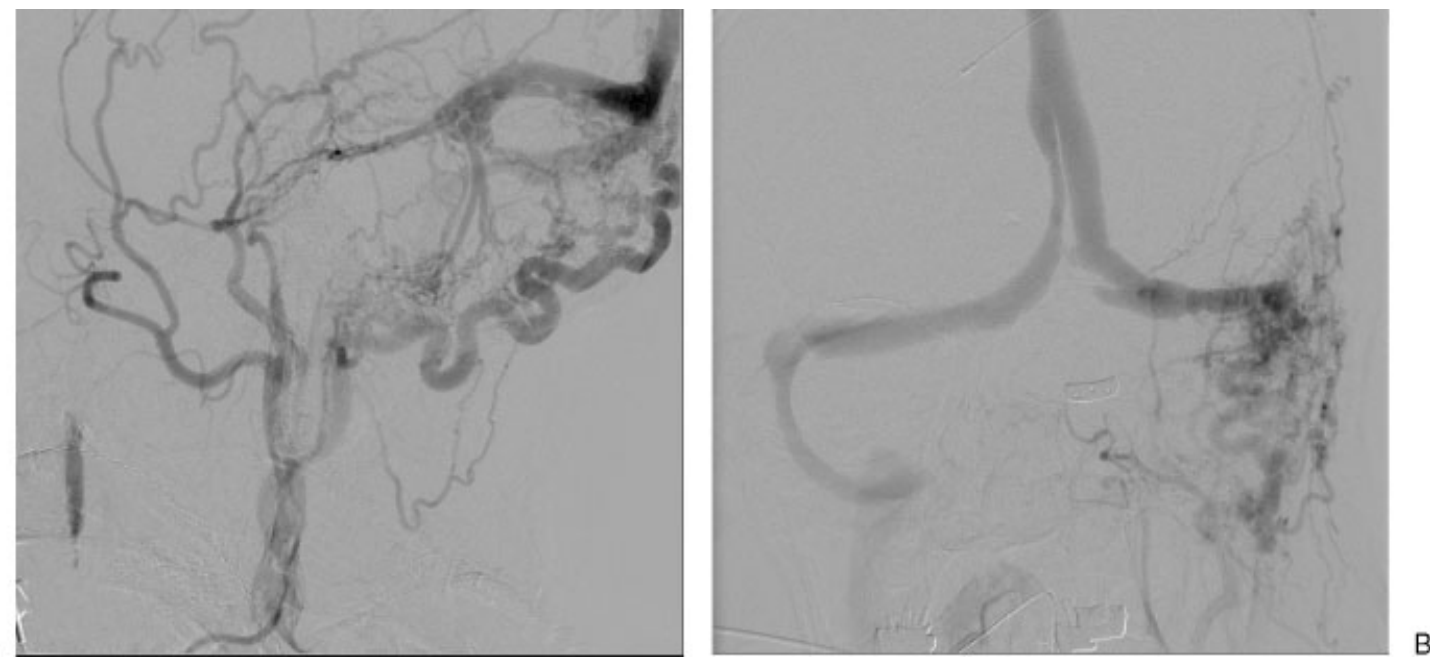

Figure 8 Patient 2: Catheter angiogram. Selective angiogram of the left external carotid artery in lateral (A) and anteroposterior (B) projections. (A) Late venous phase of an external carotid artery (ECA) injection. The left sigmoid sinus is occluded at the transverse-sigmoid junction, multiple dilated cortical veins are present, and dural sinus venous egress is in a retrograde fashion via the right transverse sigmoid sinus. (B) Arterial phase of an ECA injections demonstrate dural arteriovenous fistula at the left transverse sigmoid sinus junction, with feeders from prominent transosseous branches of the occipital artery, middle meningeal artery, and posterior auricular artery.

2 years following VS resection. ${ }^{10}$ Sakaki et al reported five cases of DAVF that developed in the transversesigmoid sinus after surgical resection of the sigmoid sinus during tumor removal, but none of the cases reported involved CPA surgery or resulted from sinus retraction alone. ${ }^{9}$

Our patients had no identifiable predisposing risk factors that would have predicted the development of DAVF. One had nonspecific symptoms, and the other was asymptomatic. Classic pulsatile tinnitus was absent, presumably related to preexisting hearing loss. Initial imaging findings were subtle and were only detected as the process evolved on follow-up imaging studies. This highlights the fact that the diagnosis of DAVF can be somewhat difficult to make on cross-sectional imaging studies, and the treating clinician and interpreting radiologist must have a high index of suspicion to make this diagnosis. This is especially true in the setting of prior posterior fossa surgery where venous sinuses may have been injured and thrombosed.

Digital subtraction catheter angiography is generally necessary to confirm the diagnosis of DAVF. Computed tomography (CT) and MRI lack sensitivity in detection of DAVFs, though recent advances in magnetic resonance and $\mathrm{CT}$ angiography have improved the noninvasive diagnosis of this entity. In a review of 46 patients with DAVF diagnosed between 1990 and 2006, Cohen et al reported that CT angiography had a sensitivity of $15.4 \%$ and MR angiography had a sensitivity of $50 \%$ compared with conventional angiography. ${ }^{13}$ Catheter angiography should therefore be performed when there is high clinical suspicion for a $\mathrm{DAVF}$, even if CT or MRI is interpreted as normal.
Endovascular therapy with embolization is the initial treatment of choice for symptomatic and progressive DAVFs involving the sigmoid or transverse sinus. ${ }^{14}$ Our cases suggest that although this can be curative, ongoing clinical and imaging vigilance is needed given the potential for these lesions to recur.

\section{CONCLUSION}

$\mathrm{DAVF}$ is an uncommon complication of CPA surgery. Difficulty in diagnosis may occur because of nonspecific presenting symptoms and subtle findings on routine postoperative imaging studies, and the potential lack of pulsatile tinnitus. Cerebral angiography is the gold standard for diagnosis, and endovascular occlusion is usually the treatment of choice. Because of the potential for considerable morbidity, every effort should be made to minimize injury to the dural sinuses during skull base surgery.

\section{REFERENCES}

1. Slattery WH III, Francis S, House KC. Perioperative morbidity of acoustic neuroma surgery. Otol Neurotol 2001; 22:895-902

2. Sanna M, Taibah A, Russo A, Falcioni M, Agarwal M. Perioperative complications in acoustic neuroma (vestibular schwannoma) surgery. Otol Neurotol 2004;25:379-386

3. Sade B, Mohr G, Dufour JJ. Vascular complications of vestibular schwannoma surgery: a comparison of the suboccipital retrosigmoid and translabyrinthine approaches. J Neurosurg 2006;105:200-204

4. Roberson JB Jr, Brackmann DE, Fayad JN. Complications of venous insufficiency after neurotologic-skull base surgery. Am J Otol 2000;21:701-705 
5. Aboian MS, Daniels DJ, Rammos SK, Pozzati E, Lanzino $\mathrm{G}$. The putative role of the venous system in the genesis of vascular malformations. Neurosurg Focus 2009;27:E9

6. Houser OW, Campbell JK, Campbell RJ, Sundt TM Jr. Arteriovenous malformation affecting the transverse dural venous sinus-an acquired lesion. Mayo Clin Proc 1979;54: 651-661

7. Shah SB, Lalwani AK, Dowd CF. Transverse/sigmoid sinus dural arteriovenous fistulas presenting as pulsatile tinnitus. Laryngoscope 1999;109:54-58

8. Awad IA, Little JR, Akarawi WP, Ahl J. Intracranial dural arteriovenous malformations: factors predisposing to an aggressive neurological course. J Neurosurg 1990;72:839-850

9. Sakaki T, Morimoto T, Nakase H, Kakizaki T, Nagata K. Dural arteriovenous fistula of the posterior fossa developing after surgical occlusion of the sigmoid sinus. Report of five cases. J Neurosurg 1996;84:113-118
10. Tsutsumi K, Shiokawa Y, Kubota M, Aoki N, Mizutani H. Postoperative arteriovenous fistula between the middle meningeal artery and the sphenoparietal sinus. Neurosurgery 1990;26:869-871

11. Desal HA, Lee SK, Kim BS, Raoul S, Tymianski M, TerBrugge KG. Multiple de novo vascular malformations in relation to diffuse venous occlusive disease: a case report. Neuroradiology 2005;47:38-42

12. Ohata K, Haque M, Morino M, et al. Occlusion of the sigmoid sinus after surgery via the presigmoidal-transpetrosal approach. J Neurosurg 1998;89:575-584

13. Cohen SD, Goins JL, Butler SG, Morris PP, Browne JD. Dural arteriovenous fistula: diagnosis, treatment, and outcomes. Laryngoscope 2009;119:293-297

14. Lv X, Jiang C, Li Y, Yang X, Wu Z. Intraarterial and intravenous treatment of transverse/sigmoid sinus dural arteriovenous fistulas. Interv Neuroradiol 2009;15:291-300 